\title{
Mudanças climáticas e ensino superior: a combinação entre pesquisa e educação
}

\section{Climatic changes and higher education: a combination between research and education}

\author{
Pedro Roberto Jacobi ${ }^{1}$
}

\begin{abstract}
RESUMO
No ensino superior observa-se o início de uma combinação original entre educação e pesquisa ligada às mudanças climáticas em programas de ciência e tecnologia. Neste início, observa-se o desafio de adaptar e desenvolver programas educativos que incluam temas associados com a diversidade regional; reconhecendo os ecossistemas complexos e diversificados, e o predomínio de um modo de vida urbano com todas as suas contradições, ligado ao consumo e, principalmente, ao aumento de uma lógica de insustentabilidade. Diante disso, comportamentos, atitudes sustentáveis e valores éticos têm de ser estimulados e compartilhados. Como encontrar alternativas educativas para abordar um tema cujos cenários são negativos e problemáticos, como indicam os relatórios do Painel Intergovernamental sobre Mudanças Climáticas - IPCC, sem cair num ponto de vista catastrofista de imobilismo ou, por outro lado, numa visão simplista a respeito de uma questão tão importante e crucial à sociedade contemporânea? Este artigo analisa a alternativa metodológica para a inclusão da temática das mudanças climáticas na formação acadêmica proposta pelo Núcleo de Apoio à Pesquisa - Mudanças Climáticas da Universidade de São Paulo (USP). Observou-se a sua adequação ao papel da educação para a promoção de aprendizagem social, construída ambientalmente - referente a processos cujos conteúdos e ênfase voltam-se à capacitação social - dentro de uma base cooperativa próxima ao pensamento crítico e à habilidade para resolução de problemas cujo foco nas necessidades auxilia as pessoas a tratar um futuro de mudanças incertas.
\end{abstract}

Palavras-chave: mudanças climáticas; ensino superior; Antropoceno; sociedade de risco; aprendizagem social.

DOI: $10.1590 / 0104-4060.38107$

1 Universidade de São Paulo. São Paulo, São Paulo, Brasil. Faculdade de Educação e Programa de Pós-Graduação em Ciência Ambiental do Instituto de Energia e Ambiente. Av. da Universidade, $\mathrm{n}^{\circ}$ 308. Cidade Universitária. CEP: 05508-040. 


\begin{abstract}
It is to be observed at the level of higher education, an original combination between research and education linked to climate changes in programs of science and technology. At this beginning, there is a need to observe the challenge to adapt and develop educational programs that include themes associated to regional diversity, recognizing complex and diversified ecosystems and the prevalence of an urban way of life with all its contradictions, linked to consumption, and mainly to an increase of a logic of unsustainability. Facing this, behaviors, sustainable attitudes and ethic values have to be stimulated and shared. How to find educational alternatives to approach a theme whose scenarios are negative and problematic, as the Intergovernmental Panel on Climate Change (IPCC) reports indicate, without falling into catastrophist immobility worldviews, or on the other side into a simplistic view related to such an important and crucial issue for contemporary society? This article analyses the methodological alternative to include the theme of climatic changes in the academic background proposed by the Sao Paulo University (USP) INterdisciplinary CLimate INvEstigation Center (INCLINE). It has been observed its adequacy to the role of education to promote social learning, environmentally built, related to processes whose contents and emphasis are centered on social capacity building - within a cooperative perspective that approaches critical thought and the ability to solve problems whose focus in needs aids people to deal with a future of uncertain changes.
\end{abstract}

Keywords: climatic changes; higher education; Anthropocene; risk society; social learning.

\title{
Um olhar sobre o planeta Terra hoje
}

As mudanças climáticas e o aquecimento da Terra indicam que estamos vivendo uma nova era glacial denominada de Antropoceno. A ação humana na natureza está promovendo alterações de grande escala na superfície terrestre há pelo menos um século. A compreensão dos fatores determinantes dos padrões climáticos mundiais desafia tanto os pesquisadores especializados como a população em geral, sobretudo devido às recentes conclusões do Painel Intergovernamental sobre Mudanças Climáticas (IPCC, 2014). O IPCC projeta um cenário radical que afetará a Terra inteira, considerando a polêmica hipótese de ser o dióxido de carbono $\left(\mathrm{CO}_{22}\right)$ a principal causa do aquecimento. Haverá eventos climáticos extremos, mudanças de ecossistemas, ascensão do nível do 
mar, migração de populações, desaparecimento de geleiras de altitude, redução das calotas polares e alterações da disponibilidade de recursos.

O aquecimento global causado pelo aumento da concentração dos gases de efeito estufa na atmosfera, devido a ações antrópicas, influencia temperaturas e circulações oceânicas, que por sua vez atuam para modificar circulações atmosféricas que terão um impacto no funcionamento dos ecossistemas. Em particular, a precipitação pode ser alterada em várias regiões do globo, afetando o manejo da água de forma geral e, com isso, a agricultura, energia e atividades socioeconômicas nas grandes cidades. Toda esta interação tem um custo socioeconômico que pode ser avaliado e posteriormente associado à vulnerabilidade das populações.

A degradação constante do ambiente tem incidido em profundas interferências na capacidade de suporte dos ecossistemas do planeta. De acordo com Rockström et al. (2009), os impactos antropogênicos vêm transgredindo limites seguros de operação global, como no caso do ciclo global de nitrogênio, da taxa de perda de biodiversidade e das mudanças climáticas. O entendimento de que o planeta está entrando em uma nova época geológica, proposta sob a denominação de Antropoceno, em que as ações humanas se constituem como a força dominante de mudanças da biosfera (CRUTZEN, 2002), indica os impactos antropogênicos nos ecossistemas como resultado do processo de busca de recursos materiais e melhores condições de vida para crescentes populações. O impacto se verifica na crescente alteração da capacidade de provimento de serviços ecossistêmicos, os quais são essenciais no suporte à qualidade de vida dos humanos. A "Era do Antropoceno" é a expressão que foi popularizada pelo geoquímico holandês Paul Crutzen, Prêmio Nobel de Química em 2002, para determinar as mudanças no planeta ocasionadas pelo homem a partir do início da Revolução Industrial. Dentre os principais elementos por trás do risco, destaca-se uma população mundial que deve subir dos atuais 7 bilhões para 9 bilhões em meados do século, mas também o consumo ineficiente e danoso de recursos, e o que um número cada vez maior de cientistas afirma é que no prazo de poucas décadas, muitos ecossistemas vitais poderiam sofrer danos duradouros ou irreversíveis por causa do aquecimento provocado pelo homem.

O tema das alterações climáticas está se transformando em algo que supera as dimensões de um problema ambiental. As mais importantes sociedades científicas são cada vez mais unânimes em afirmar que a humanidade tornou-se a principal força de mudança geológica do planeta e a capacidade do planeta para continuar assimilando e atenuando os impactos vindos da pressão humana está dando visíveis sinais de esgotamento. O grupo liderado por Johan Rockström (2009) indica a necessidade de definição dos limites planetários, e coloca a ques- 
tão sobre o espaço seguro de operação cuja ultrapassagem impede que o planeta continue oferecendo os serviços ecossistêmicos que, até aqui, têm permitido o processo de desenvolvimento.

Os autores expõem outros quatro processos e definem seus limites: perda de biodiversidade, uso de água fresca, mudanças no uso da terra e ciclos do nitrogênio e do fósforo. A situação de transgressão dos limites seguros de operação global vem a estabelecer o imperativo de funções socioecológicas de resiliência para atenuar os efeitos combinados das mudanças ambientais. No entanto, todas as possibilidades inerentes à resiliência, mitigação e adaptação frente às mudanças climáticas requerem, primeiramente, o reconhecimento de toda a sociedade, tanto para o que diz respeito a ações pertinentes em nível individual quanto da comunidade, regional, nacional ou internacional. Para a tomada de decisões, estabelecimento de agendas e de ações, nesse sentido, o primeiro requisito, é, indubitavelmente, a percepção do problema e de sua relevância. Na verdade, a percepção deve abranger um sentido amplo das mudanças climáticas e de seus desdobramentos, que se ampliam em escalas temporais e espaciais.

A ciência avançou na identificação de fronteiras planetárias dentro das quais a humanidade poderia operar de forma segura em referência ao funcionamento do sistema terrestre. Atravessar essas fronteiras implicaria entrar numa zona de risco de disrupção ambiental sistêmica. Dessa forma, nove fronteiras planetárias são identificadas, sete das quais são passíveis de serem quantificadas: mudança climática; acidificação dos oceanos; ozônio; ciclo biogeoquímico do nitrogênio e fósforo; uso da água doce; mudanças no uso da terra; biodiversidade; poluição química; e concentração de aerossóis na atmosfera. Três dessas nove fronteiras planetárias já foram ultrapassadas: mudança climática, taxa de perda de biodiversidade e ciclo do nitrogênio.

Cabe destacar que as mudanças globais anunciadas pelo Antropoceno serão graduais ou que, uma vez manifestados seus efeitos, possa ser simples a sua reversão, interferindo nas suas causas. Uma das mais importantes contribuições científicas que se tornou parte do argumento central está associada com o conceito de Resiliência, que implica na capacidade de um sistema (um indivíduo, uma floresta, uma cidade ou uma economia) lidar com a mudança incremental ou abrupta e prosseguir em seu desenvolvimento. O que as pesquisas têm demonstrado é que os sistemas, longe de mudarem de forma contínua e gradativa, conhecem mudanças bruscas, inesperadas e, muitas vezes, irreversíveis. Na abordagem de Giddens (2010), as mudanças climáticas demandam uma ação contundente, contínua e multissetorial na qual o Estado seja o grande motivador e assegurador, no sentido de viabilizar e estimular os setores da sociedade mais atuantes. Visto que os perigos representados pelo aquecimento global não são 
palpáveis, imediatos ou visíveis no decorrer da vida cotidiana, os avanços têm sido muito lentos, associados com um quadro global que demanda grandes transformações, e no qual as mudanças climáticas estão deixando de ser uma preocupação fundamentalmente ecológica ou ambiental e passam a ser um fator decisivo do próprio cálculo dos mais importantes atores econômicos globais.

Deve destacar-se a dimensão da governança global que permite considerar a grande quantidade de interações sociais que contornam as fronteiras da política global de clima. Trata-se de amplo conjunto de atores, localizados em vários níveis - nacionais, subnacionais, internacionais, transnacionais -, que interagem em diferentes âmbitos, portadores de uma diversidade de interesses e motivações. Por outro lado, a economia política da mudança climática introduz as consequências do fenômeno sobre a estrutura econômica global em duas dimensões: os custos dos efeitos do aquecimento global sobre a vida econômica das sociedades e os custos de transição para uma economia de baixo carbono. Essa área de conhecimento considera a estrutura de incentivos econômicos que guia a decisão dos atores envolvidos na dinâmica global de clima. Um terceiro aspecto relevante está associado com a segurança climática e os desafios colocados para pensar a interação entre a mudança climática e as relações internacionais, tanto no plano dos efeitos da desestabilização climática sobre certas pautas de conflito e cooperação em vários níveis da governança quanto no entendimento de como a forma dos interesses de segurança podem afetar a consolidação de uma estrutura eficaz de governança global sobre clima (VIOLA; FRANCHITTI, 2012).

Para Giddens (2010), o Estado assegurador deve contribuir para que os governos desenvolvam uma ação antecipatória, o que muito pouco tem acontecido, incentivando as empresas, o terceiro setor e o cidadão - em termos de políticas a longo prazo - a enfrentar os riscos da mudança climática e da energia em relação a outros riscos enfrentados pelas sociedades atuais. Diversas ações se tornam estratégicas; como a convergência política e econômica entre a mudança climática e a política energética, assim como avanços na estrutura econômica e fiscal em direção a uma economia com baixo teor de carbono, e também colocar em xeque os interesses empresariais que dificultam ou impedem iniciativas ligadas à mudança climática; fortalecer o tema da mudança climática na agenda política; e finalmente ampliar diálogos entre ciência, governos e sociedade e capacitar atores sociais dos diferentes segmentos, público e privado, para promover ações e políticas de adaptação às consequências da mudança climática e integrar os aspectos locais, regionais, nacionais e internacionais da política de mudança climática. 


\section{A necessidade de ruptura com a compartimentação do conhecimento}

Nesse contexto, ao passo em que se ressalta a complexidade dos eventos e a necessidade de diálogo entre ciência, gestores e sociedade, chama atenção a prevalência de uma racionalidade cognitivo-instrumental que tem agravado a situação ambiental do planeta, mantendo uma relação abissal tanto quanto às desigualdades materiais como no que concerne à diversidade de saberes, muitos dos quais, embora marginalizados, apresentam-se com elevado poder de aplicação para os desdobramentos locais oriundos de processos globais (SANTOS, 2007).

Essa busca de respostas na interdisciplinaridade deve-se à constatação de que os problemas que afetam e mantêm a vida no nosso planeta são de natureza global e de que suas causas não podem restringir-se apenas aos fatores estritamente biológicos: pois revelam dimensões políticas, econômicas, institucionais, sociais e culturais.

O desafio da interdisciplinaridade implica num processo de conhecimento que busca estabelecer cortes transversais na compreensão e explicação do contexto da pesquisa. Busca-se a interação entre disciplinas, superando-se a compartimentação científica provocada pela excessiva especialização. Enquanto combinação de várias áreas de conhecimento, a interdisciplinaridade pressupõe o desenvolvimento de metodologias interativas, configurando a abrangência de enfoque.

A preocupação em consolidar uma dinâmica de ensino e pesquisa com base em uma perspectiva interdisciplinar enfatiza a importância dos processos sociais que determinam as formas de apropriação da natureza e suas transformações através da participação social na gestão dos recursos ambientais, levando em conta a dimensão evolutiva no sentido mais amplo, incluindo as conexões entre as diversidades biológica e cultural, assim como as práticas dos diversos atores sociais, bem como o impacto da sua relação com o meio ambiente.

Para tanto, coloca-se o desafio de ruptura com a compartimentação do conhecimento e marginalização da diversidade de saberes, e isto envolve um conjunto de atores do universo educativo em todos os níveis. Abre-se a possibilidade de potencializar outras racionalidades para o engajamento dos diversos sistemas de conhecimento, da formação e profissionalização docente, de profissionais em geral e da comunidade universitária, fortalecendo conteúdos e conhecimento baseados em valores e práticas sustentáveis, indispensáveis para estimular o interesse, o engajamento e a responsabilização.

A multiplicação dos problemas ambientais tem imposto às diversas disciplinas científicas temas para os quais estas não estavam anteriormente 
preparadas e para cujo enfrentamento são obrigadas a reformular os parâmetros de ensino e pesquisa.

Os riscos contemporâneos (BECK, 2010) explicitam os limites e as consequências das práticas sociais, trazendo consigo um novo elemento, a "reflexividade". Para Beck (2007), viver numa sociedade de risco significa que a controlabilidade dos efeitos colaterais e dos perigos produzidos pelas decisões tornou-se problemática, e os saberes podem servir para transformar os riscos imprevisíveis em riscos calculáveis, e a indeterminabilidade do risco no presente se torna, pela primeira vez, fundamental para toda a sociedade. Assim, sem renunciar às especialidades disciplinares, a noção do meio ambiente recoloca o ser humano no centro das preocupações e dos programas científicos.

As transformações sociais em curso demandam cada vez mais concepções interdisciplinares para orientar estratégias de pesquisa e de formação de políticas ambientais e de desenvolvimento sustentável, devendo-se reconhecer os efeitos das políticas econômicas vigentes sobre a dinâmica dos ecossistemas e sobre as condições de vida das sociedades (FUNTOWICZ, 2000). A visão de complexidade representa a resposta face às articulações despedaçadas pelos cortes entre disciplinas, entre categorias cognitivas e entre tipos de conhecimento (MORIN, 1998). Os enfoques de conhecimento se consolidam tendo como referentes os estudos em torno dos efeitos da problemática ambiental sobre as transformações metodológicas, os diálogos interdisciplinares que abrem um novo horizonte para o diagnóstico das mudanças socioambientais, rompendo com o reducionismo e abrindo espaço para uma realidade permeada por incertezas, o que propicia a formulação de diferentes abordagens em torno da sustentabilidade ambiental (JACOBI, 2012).

A ênfase em práticas que estimulam a interdisciplinaridade e a transversalidade revela o grande potencial que existe para sair do lugar comum e o trabalho com temáticas que incitam mudanças no comportamento, na responsabilidade socioambiental e na ética ambiental, o que estimula outro olhar. Trata-se da importância de compreender a complexidade envolvida nos processos e o desafio de ter uma atitude mais reflexiva e atuante e, por conseguinte, que os cidadãos se tornem mais responsáveis, cuidadosos e engajados em processos colaborativos com o meio ambiente no contexto da sociedade de risco (WALS, 2007).

Assim, a multiplicação dos problemas ambientais tem imposto às diversas disciplinas científicas temas para os quais estas não estavam anteriormente preparadas e para cujo enfrentamento se demanda reformular os parâmetros de ensino e pesquisa. Sem renunciar às especialidades disciplinares atualmente em vigor, mas certamente contribuindo para sua reformulação e desenvolvimento, a noção dos problemas socioambientais recoloca o ser humano no centro das preocupações e dos programas científicos. 
Neste sentido, estes diálogos interdisciplinares demandam novas formas de abordagem na relação com os atores sociais envolvidos em ações educativas nas quais, conforme De Marchi e Ravetz (1999), se evidenciam que fenômenos emergentes serão de forma crescente uma constante num cenário de complexos sistemas sociotécnicos. O grande desafio está na necessidade de dar transparência ao conteúdo em atividades educativas em diversos espaços que com foco nas questões colocadas pela sociedade de risco reforçam a necessidade de colocar em debate temas que têm, nos diferentes tipos de incerteza, a necessidade de multiplicar conhecimentos e diálogos (JACOBI; GIATTI; AMBRIZZI, 2014).

Funtowicz e Ravetz $(1993,1997)$ apresentam um método que, baseado no reconhecimento da incerteza, da complexidade e da qualidade, guia um empreendimento científico que denominam de "ciência pós-normal". Essa abordagem tem nas "comunidades ampliadas de pares", descritas por meio de grupos focais, júris de cidadãos, conferências de consenso, fóruns consultivos cujos stakeholders tenham algum grau de legitimidade e influência, atores estratégicos para estimular e legitimar o diálogo e respeito entre diferentes campos do saber e possibilitar maior qualidade e validade para o saber científico (JACOBI; GIATTI; AMBRIZZI, 2014).

Sob orientação da ciência pós-normal, ressalta-se a necessidade de superação do princípio clássico da prevenção, o qual se constitui com base em causalidades conhecidas, comprovadas. Diante de incertezas inerentes à multiplicidade de fatores e à complexidade das relações entre homem e ambiente, soma-se, ao necessário processo de prevenção primária, a proposição de uma ciência e prática da precaução (FREITAS; PORTO, 2006), que seja capaz de lidar com planos de ação e orientá-los diante de ocorrências inesperadas. Essa configuração, de maior inter-relação entre ciência, sociedade e processos políticos, por sua vez, não é capaz de ampliar o grau de confiança no aparato preditivo científico. Por seu turno, os avanços tangíveis nesse sentido constituem-se pelo reconhecimento das incertezas e pelo avanço em escolhas consensuais que prezem pela prudência, pela precaução, em detrimento de escolhas permeadas por elevado grau de incertezas, como no caso de novas tecnologias que possam trazer riscos muito mais elevados do que as perspectivas de um ganho social com equidade.

Observa-se, portanto, que o saber complexo demanda avanços nas fronteiras disciplinares, o que Raynaut (2011, p. 84) qualifica como "um enfoque interdisciplinar [que] consiste em tentar restituir, ainda que de maneira parcial, o caráter de totalidade, de complexidade e de hibridação do mundo real, dentro do qual e sobre o qual pretendemos atuar".

A novidade dos objetos científicos híbridos é a ruptura de fronteiras de conhecimento, de preconceitos, de hierarquias de saberes e da desconfiança entre disciplinas. Isso deve ocorrer por meio de cortes transversais e dinâmicas 
colaborativas entre áreas de conhecimento e pela combinação de metodologias que permitam nova configuração das conexões entre as ciências naturais, sociais e exatas. O diálogo entre disciplinas e a vivência de experiências de ensino e pesquisa sob esses preceitos visam construir um campo de conhecimento capaz de captar as multicausalidades e as relações de interdependência dos processos de ordem natural e social que determinam as estruturas e mudanças socioambientais. Essa ênfase se coloca pela busca de novas formas de gerar conhecimento e de promover a inflexão na estrutura consolidada que gerou uma hierarquia de saberes (JACOBI, 2012).

O caminho para uma sociedade sustentável se fortalece na medida em que se ampliem práticas educativas que, pautadas pelo paradigma da complexidade, conduzam para uma atitude reflexiva em torno da problemática ambiental, visando traduzir o conceito de ambiente na formação de novas mentalidades, conhecimentos e comportamentos. A ênfase na abordagem da complexidade coloca-se como uma alternativa para a busca de novas formas de gerar conhecimento, e promove uma inflexão na estrutura consolidada que gerou uma hierarquia de saberes.

Nesse sentido, o trabalho intersetorial se apresenta como uma importante contribuição para estabelecer melhores condições para uma lógica cooperativa e para abrir um novo espaço, não só para a sociedade civil, mas também para os sistemas peritos (GIDDENS, 1992). A ênfase em práticas que estimulam a interdisciplinaridade e a transversalidade revela o grande potencial que existe para o trabalho com temáticas que incitam mudanças no comportamento, na responsabilidade socioambiental e na ética ambiental, o que estimula outro olhar. Trata-se da importância de compreender a complexidade envolvida nos processos e o desafio de ter uma atitude mais reflexiva e atuante e, por conseguinte, que os cidadãos se tornem mais responsáveis, cuidadosos e engajados em processos colaborativos com o meio ambiente (WALS, 2007).

As experiências e práticas educativas e de pesquisa interdisciplinares ainda são recentes e incipientes. Os processos de conhecimento buscam estabelecer cortes transversais na compreensão e explicação dos contextos de aprendizagem e de formação. $\mathrm{O}$ estímulo é para a interação e interdependência entre as disciplinas e, consequentemente, entre as pessoas, para o desenvolvimento de metodologias interativas. Aborda-se o estudo e conhecimento do meio ambiente através de uma concepção integrada, interdisciplinar, identificando disponibilidades e avaliando consequências do uso dos recursos naturais, fontes energéticas, tecnologias e empreendimentos, na formação do conhecimento e nas práticas e estruturas socioculturais. 


\section{Mudanças climáticas, Aprendizagem Social e governança adaptativa}

Face à imprevisibilidade das consequências das mudanças climáticas, diversas questões se colocam nos dias de hoje associadas com a necessidade de definir estratégias para enfrentar as mudanças climáticas, tornar a sociedade mais reflexiva e, portanto, mais resiliente aos efeitos diretos e indiretos das mudanças climáticas, sensibilizar e criar condições para promover ações pautadas pelo reconhecimento dos riscos e incluir a temática das mudanças climáticas na formação acadêmica.

A relação entre formações de quadros profissionais nas áreas pública e privada que tenham maior compreensão e domínio dos aspectos que compreendem o enfrentamento dos riscos se torna determinante para fazer frente à magnitude dos eventos naturais adversos, assim potencializando a redução da vulnerabilidade das comunidades e, portanto, minimizando a intensidade dos desastres e de riscos indiretos que interagem de forma sistêmica com outros aspectos ambientais e sociais em distintas escalas espaciais e temporais. Para isso, avanços interdisciplinares na forma de diálogo entre saberes acadêmicos e sociedade podem fazer com que os mais distintos atores sociais, inclusive na qualidade de sujeitos dos riscos, possam se apropriar de elementos das inter-relações entre variabilidade climática regional e outros problemas socioambientais, incluindo a saúde humana.

Os riscos associados às mudanças climáticas e ambientais implicam em ampla revisão das práticas de governança dos riscos como estratégia de enfrentamento dos problemas, fortalecendo-se os processos de governança adaptativa, o que requer que os processos decisórios sejam abertos e participativos. Isto demanda novas estratégias, que pautadas pelo diálogo, impliquem em políticas para enfrentar problemas e riscos com a ampliação dos atores envolvidos de forma a contribuir com seu conhecimento para complementar o conhecimento técnico.

A adaptação à mudança climática global e a transição para a economia verde demandarão novas capacitações, em particular, novas especialidades ocupacionais, modos de aprendizado, gestão e maior esforço de pesquisa. Todo o sistema de educação e treinamento, no mundo todo, terá que mudar, para atender a esta nova demanda. Hoje já há falta de recursos humanos especializados em muitas das áreas emergentes da economia verde e na capacitação para a formulação de propostas alternativas e inovadoras tanto na gestão pública quanto no setor privado (JACOBI; SINISGALLI, 2012).

Para tanto, quanto mais as ações de educação ambiental dialogarem com visões pautadas pela existência de riscos promovidos pela sociedade humana, 
denominados de efeitos antrópicos, maiores serão as possibilidades de formar atores sociais mobilizadores e multiplicadores nos diversos setores da sociedade. No entanto, para quebrar o hiato existente entre o reconhecimento da crise social e ambiental e a construção real de práticas capazes de estruturar as bases de uma sociedade sustentável, coloca-se a necessidade de fortalecimento de comunidades de prática (WENGER, 1998) e da Aprendizagem Social (GLASSER, 2007).

Os referenciais da Aprendizagem Social se inserem nas práticas socioambientais educativas de caráter colaborativo, que têm se revelado como veículo importante na construção de uma nova cultura de diálogo e participação (JACOBI et al., 2006). Como práxis educativa engajada e política, abre um estimulante espaço para a construção de eixos interdisciplinares em torno dos quais se tece uma nova cultura para a formação abrangente, a partir de uma abordagem sistêmica e complexa.

Trata-se de abordagem integradora das relações entre as esferas subjetivas e intersubjetivas que amplia a possibilidade de constituição de identidades coletivas em espaços de convivência e debates.

O maior desafio é criar oportunidades de aprendizagem social ativas, nas quais haja o real envolvimento dos sujeitos em relações de diálogo que favoreçam: a percepção da diversidade de opiniões e visões de mundo; a mediação de interesses individuais e coletivos; e a possibilidade de ampliação de repertórios que aumentem a capacidade de contextualizar e refletir (GLASSER, 2007).

As práticas pautadas pelo conceito de Aprendizagem Social pretendem, portanto, integrar os seguintes fatores: uma reflexão crítica; o desenvolvimento de um processo participativo, múltiplo e democrático; a construção de uma percepção partilhada do problema em relação ao grupo de atores sociais envolvidos; e o reconhecimento das interdependências e das interações dos atores (PAHL-WOSTL, 2002; JIGGINS, 2007).

\section{Novos caminhos para práticas cooperativas e interdisciplinares face às mudanças climáticas}

As questões associadas às mudanças climáticas e seus potenciais desdobramentos, afetando a saúde e qualidade de vida dos humanos, representam tema de elevada seriedade, permeado por um quadro de incertezas. E a ampliação das vulnerabilidades e a necessidade de administração dos riscos ambientais, principalmente das mudanças climáticas, e a distribuição desigual de riscos, aspectos de elevada complexidade, nos mostram que estão em jogo valores 
controvertidos, fatos incertos, necessidade de ações urgentes e elevados conflitos de interesse. Isto nos leva a considerar que há iminente necessidade de que todas as partes interessadas, como os diversos atores sociais, ampliem sua consciência crítica e reflexiva, no sentido de controlar criticamente a relação da ciência com a gestão dos riscos, estabelecendo bases para uma ciência precaucionária (RAVETZ, 2004).

Ao enfatizar o conceito de Aprendizagem Social, se entende que os diversos atores sociais envolvidos possam ampliar, diversificar e hibridizar seus conhecimentos por meio de caminhos e práticas interdisciplinares, estabelecendo laços de confiança e cooperação. Ao se promover um diálogo entre ciência, sociedade e gestão, se criam as condições para a necessária compreensão sobre as complexidades inerentes às mudanças climáticas e avanços em torno da problemática socioambiental, na formação de novas mentalidades, conhecimentos, valores e comportamentos.

Um dos maiores desafios é o de criar oportunidades de aprendizagem social ativas, nas quais ocorra o envolvimento em relações de diálogo e de ampliação de repertórios que aumentem a capacidade de contextualizar e refletir. Os processos coletivos, principalmente os de base cooperativa, possuem um potencial para a emergência de inovações de compromissos coletivos e de práticas de cidadania orientadas para a sustentabilidade (JACOBI, 2012).

Nesse sentido, a alternativa para a inclusão da temática das mudanças climáticas na formação acadêmica na Universidade de São Paulo, numa perspectiva interdisciplinar, interligando diferentes equipes e projetos de pesquisa de áreas distintas do conhecimento, se desenvolve num contexto no qual o pensamento teórico se prolonga em uma prática científica para gerar conhecimento. Face à natureza complexa, indo além do pensamento racional científico e da utilização de seus instrumentos conceituais e metodológicos, assume-se uma postura intelectual frente à natureza complexa, estabelecendo relações e estando em permanente reconstrução frente às interferências das ações humanas sobre o ambiente que atingiram escala global e magnitude sem precedentes, afetando o funcionamento natural do sistema climático.

Neste contexto, com apoio da Universidade de São Paulo (USP), em 2011 foi criado o Núcleo de Apoio à Pesquisa - Mudanças Climáticas (em inglês, "INterdisciplinary CLimate INvEstigation Center - INCLINE"), que visa articular os diversos atores no campo acadêmico e científico dentro da Universidade para ampliar os diálogos entre professores, pesquisadores, alunos de pós-graduação e iniciação científica em torno de um tema fundamental para a USP, para o Brasil e para o planeta, prestando apoio a órgãos governamentais e atuando como interlocutor entre a pesquisa científica desenvolvida dentro da universidade e a sociedade. A partir dos 12 Programas de Pós-Graduação 
que agregam 16 grupos de pesquisa e mais de 50 pesquisadores da USP e suas articulações externas, tem-se como objetivo desenvolver modelos mais detalhados sobre o comportamento climático no Brasil, visando uma atuação em conjunto com a iniciativa pública na tentativa de realizar ações mais eficientes nas questões que tangem o clima.

Além do papel estritamente técnico de produzir conhecimento e pesquisa nova, um núcleo como esse tem outro papel importante: que o grupo se torne um "polo agregador na temática de mudanças climáticas", tornando-se referência nacional e internacional no assunto, sintetizando e avaliando informações científicas sobre os aspectos relevantes das mudanças climáticas no Brasil, e dessa forma, auxiliando os formuladores de políticas públicas e a sociedade em geral, que necessitam cada vez mais de informações objetivas sobre as causas das mudanças climáticas, seus impactos ambientais e socioeconômicos e as possíveis soluções.

A inter-relação dos diferentes aspectos e impactos das mudanças climáticas e sua interdisciplinaridade dentro do INCLINE visa promover um maior conhecimento da variabilidade climática atmosférica global e da América do Sul, juntamente com os oceanos, aliado a um entendimento mais profundo do papel da floresta amazônica no contexto do clima global e regional, além das mudanças regionais, onde a discussão da bioenergia pode causar um impacto na agricultura e onde as grandes cidades sofrem com o aumento da poluição e do calor, sendo também mais vulneráveis a eventos extremos de tempo, causando danos econômicos e perdas de vida, além de impactos à saúde.

Por meio do aprofundamento de pesquisas e atividades pautadas pela interdisciplinaridade (RAYNAUT, 2010), o núcleo poderá contribuir para o melhor entendimento dos complexos sistemas de interações entre Terra-Ambiente-Homem pelo desenho e implantação de projeto de natureza interdisciplinar, agregando especialistas de diversas áreas e fazendo as necessárias conexões com os formuladores de políticas públicas e a iniciativa privada, cumprindo a sua função de propor soluções e alternativas viáveis para a redução das emissões, mitigação dos atuais problemas enfrentados e apontando novas direções e modelos de crescimento sustentável.

Entretanto, o maior desafio para o Núcleo é o de criar um ambiente de trabalho interdisciplinar. Para tanto, com base nas premissas de uma estratégia de fortalecer Aprendizagem Social, torna-se desafio que todos os envolvidos consigam descobrir seu espaço na pesquisa e é importante ter uma sólida formação disciplinar e, ao mesmo tempo, que seja multidisciplinar. Uma das premissas é de também desenvolver uma estrutura conceitual e analítica comum, tendo uma metodologia interdisciplinar e constituindo uma base epistemológica comum. Além disso, enfatiza-se a sistematização das discussões em grupo e 
que todos participem do processo formativo sobre conceitos e metodologias, criando interfaces de análise e método. Todos estes conceitos têm sido aplicados dentro do Núcleo através de reuniões gerais do grupo, elaboração de oficinas para definição de conceitos de uso comum no Núcleo e participação em projetos pedagógicos interdisciplinares.

\section{REFERÊNCIAS}

BECK, Ulrich. World at Risk. Cambridge: Polity Press, 2008.

BECK, Ulrich. Sociedade de Risco. São Paulo: Editora 34, 2010.

CRUTZEN, Paul. J. Geology of mankind: the Anthropocene. Nature, v. 415, p. 23, 2002.

DE MARCHI, Bruna; RAVETZ, Jerome. Risk Management and Governance: a PostNormal Science Approach. Futures, v. 31, n. 7, p. 743-757, Sept. 1999.

FREITAS, Carlos. M.; PORTO, Marcelo, F. Saúde, ambiente e sustentabilidade. Rio de Janeiro: Fiocruz, 2006.

FUNTOWICZ, Silvio; RAVETZ, Jerome. La ciencia posnormal. Barcelona: Icaria, 2000.

FUNTOWICZ, Silvio; RAVETZ, Jerome. Ciência Pós-normal e comunidades ampliadas de pares face aos desafios ambientais. História, Ciência, Saúde, v. 4, n. 2, p. 219-230, 1997.

FUNTOWICZ, Silvio; RAVETZ, Jerome. Science for the Post-Normal Age. Futures, v. 25, p. 735-755, 1993.

GLASSER, Harold. Minding the gap - the role of social learning in linking our stated desire for a more sustainable world to our everyday actions and policies. In: WALS, Arjen (Ed.). Social learning: towards a sustainable world. Wageningen: Wageningen Academic Publishers, 2007.

JACOBI, Pedro Roberto; GIATTI, Leandro; AMBRIZZI, Tercio. Interdisciplinaridade e mudanças climáticas: caminhos de reflexão para a sustentabilidade. In: PHILIPPI, Arlindo; FERNANDES, Valdir (Eds.). Práticas de Interdisciplinaridade no ensino e na pesquisa. São Paulo: Manole, 2014. Cap. 13.

JACOBI, Pedro Roberto. Governança ambiental, participação social e educação para a sustentabilidade. In: PHILIPPI, A. et al. (Eds.). Gestão da Natureza Pública e Sustentabilidade. São Paulo: Manole, 2012.

JACOBI, Pedro Roberto; SINISGALLI, Paulo. Governança ambiental e economia verde. Ciência \& Saúde Coletiva [online], v. 17, n. 6, p. 1469-1478, 2012. 
JACOBI, Pedro Roberto; TRISTÃO, Martha; FRANCO, Maria Isabel. A função social da educação ambiental nas práticas colaborativas: participação e engajamento. Caderno Cedes, v. 29, n. 77, jan./abr. 2009.

JACOBI, Pedro Roberto; FRANCO, Maria Isabel; GRANJA, Sandra Ines. Aprendizagem Social: práticas educativas e participação da sociedade civil como estratégias de aprimoramento para a gestão compartilhada em bacias hidrográficas. São Paulo em Perspectiva, v. 20, n. 2, 2006.

JIGGINS, Janice et al. Social learning in situations of competing claims on water use. In: WALS, Arjen (Ed.). Social learning: towards a sustainable world. Wageningen: Wageningen Academic Publishers, 2007.

MORIN, Edgar. Ciência com Consciência. Rio de Janeiro: Bertrand Brasil, 2007.

MORIN, Edgar. A religação dos saberes: o desafio do século XXI. Rio de Janeiro: Bertrand Brasil, 2004.

MORIN, Edgar. A cabeça bem-feita: repensar a reforma - reformar o pensamento. Rio de Janeiro: Bertrand Brasil, 2000.

PAHL-WOSTL, Claudia et al. Social learning and water resources management. Ecology and Society, v. 12, n. 2, p. 5, 2007. Disponível em: <http://www. ecologyandsociety.org/ vol12/iss2/art5/>. Acesso em: 21/5/2010.

PAHL-WOSTL, Claudia. Towards sustainability in the water sector - The importance of human actors and processes of social learning. Aquatic Sciences, v. 64, p. 394-411, 2002.

RAVETZ, Jerome. The post-normal science of precaution. Futures, v. 36, n. 3, p. 347357. Apr. 2004.

RAYNAUT, Claude. Interdisciplinaridade: mundo contemporâneo, complexidade e desafios à produção e à aplicação de conhecimentos. In: PHILIPPI, Arlindo; SILVA NETO, Antonio (Eds.). Interdisciplinaridade em Ciência, Tecnologia e Inovação. São Paulo: Manole, 2011.

ROCKSTRÖM, Johan et al. Planetary boundaries: Exploring the safe operating space for humanity. Ecology and Society, v. 14, n. 2, 2009.

SANTOS, Boaventura Souza. Para além do pensamento abissal: Das linhas globais a uma ecologia de saberes. Novos estudos CEBRAP, n. 79, p. 71-94, 2007.

SANTOS, Vania M. Formação de professores para o estudo do ambiente: projetos escolares e a realidade socioambiental local. Tese (Doutorado) - Instituto de Geociências, Unicamp. Campinas, 2006.

STERLING, Stephen. Riding the storm: towards a connective cultural consciousness. In: WALS, Arjen (Ed.). Social learning towards a sustainable world. Wageningen: Wageningen Academic Publishers, 2007. 
TILBURY, D. Environmental Education for Sustainability: A Force for Change in Higher Education. In: CORCORAN, Peter; WALS, Arjen (Eds.). Higher Education and the Challenge of Sustainability: Problematics, Promise, and Practice. Dordrecht: Kluwer Academic Publishers, 2004.

VIOLA, Eduardo; FRANCHINI, Matias. Sistema internacional de hegemonia conservadora: o fracasso da Rio + 20 na governança dos limites planetários. Ambiente e Sociedade, São Paulo, v. 15, n. 3, set./dez. 2012.

WALS, A. Social learning: towards a sustainable world. Wageningen: Wageningen Academic Publishers, 2007.

WENGER, Etienne. Communities of practice: learning, meaning, and identity. New York: Cambridge University Press, 1998.

Texto recebido em 09 de outubro de 2014. Texto aprovado em 09 de outubro de 2014. 